\title{
Mass transfer of organic substances in supercritical carbon dioxide
}

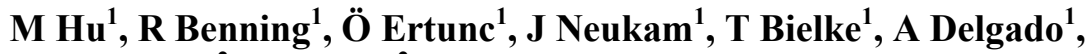 \\ V Nercissian', A Berger ${ }^{2}$ \\ ${ }^{1}$ Institute of Fluid Mechanics, Friedrich-Alexander University Erlangen-Nuremberg, \\ Cauerstrasse 4, \\ ${ }^{2}$ Institute of Optics, Information and Photonics, Friedrich-Alexander University \\ Erlangen-Nuremberg, Staudtstr.7 / B2, \\ 91058 Erlangen, Germany \\ E-mail: miao.hu@1stm.uni-erlangen.de
}

\begin{abstract}
In this work special attention is paid on the direct visualization of the diffusion process of oil droplets in supercritical carbon dioxide as well as better characterization of the process by quantitatively evaluating the important parameter - the diffusion coefficients obtained with a shearing interferometer. Experiments are also to be carried out under microgravity to improve the experiment condition where the influence of gravity-driven convection that usually dominates the transport process is minimized.
\end{abstract}

\section{Introduction}

Supercritical fluids have been widely utilized as solvents in the chemical industries, as well known in the material processing procedures, e.g. tea and coffee decaffeination, hops and flavor extraction, pharmaceuticals isolation and fractionation, etc. Supercritical fluids, especially carbon dioxide, water and alcohols have been recognized as excellent alternatives to the conventional chemical solvents in many areas, including separation, chemical reaction, surface processing and waste treatment etc. They can provide clean and sustainable chemical processes with less energy consumption and high efficiency, which keeps up with the increasing awareness of health, environment and safety issues in nutrition and medicine production processes. To achieve a better understanding of the process as well as a more precise system control of the parameters, research into supercritical fluids concerning thermodynamic fundamentals, i.e. fluid dynamics, heat and mass transfer, interfacial phenomena, etc. is very helpful and will contribute to the further development of their large - scale industrial applications [1].

Besides the well known environmental aspects of supercritical carbon dioxide $\left(\mathrm{SCCO}_{2}\right)$, it is also advantageous in the extraction processes as solvent due to its preferable solubility properties. It has been proved to be selective in the separation of desired compounds, with the relatively modified critical parameters $\left(T_{c}=304.1 \mathrm{~K}, P_{c}=7.38 \mathrm{MPa}\right)$ it does not tend to leave any toxic residuals in the extracts or to cause potential thermal degradation of the products being processed. The application of $\mathrm{SCCO}_{2}$ to extract and to isolate compounds from natural materials has been documented for nearly three decades [2]. 
Although possessing many obvious advantages, the commercial applications are still restricted to very few products due to high investment costs for this novel but unfamiliar treatment. Necessary advances in process, equipment and product design as well as the realization of the potentially economic opportunities in the market of high value products manufacturing could lead to higher interest in the investment and further development in this branch. [3]

\section{Experiment implementation}

\subsection{Concept and experiment design}

To exploit the behavior of supercritical fluids near their critical points and their transport properties, which are essential in designing reactors and optimization of the processing procedures, e.g., many efforts have been made to quantify the dissolving process by precisely measuring the transport coefficients. Well known are Taylor-Aris Dispersion (also known as Capillary Peak Broadening and Supercritical Chromatography Technique), Solid Dissolution, Photon Correlation Spectroscopy, Nuclear Magnetic Resonance (NMR), etc. [4] In this work, a new optical approach - shearing interferometer - was applied to visualize directly as well as to analyze quantitatively the phenomenon. It provides a noninvasive observation method with the possibility of being automated and integrated into the industrial scale - setups serving as the inspection or control link.

The concept was to visualize the diffusion of a sample droplet (solute) with a diameter of ca. $2 \mathrm{~mm}$ in supercritical $\mathrm{CO}_{2}$ (solvent). In this dimension, it requires great care to minimize the interference of convection, which is largely enhanced by the gravity and also by the sensitivity of supercritical fluids to small changes in temperature and pressure. In order to better control the experiment conditions, an experimental setup was specially built to provide a stable environment. Besides, experiments under microgravity (two parabolic flight campaigns) were also carried out. The proposed setup to implement the experiments is shown in Figure 2.1 below.

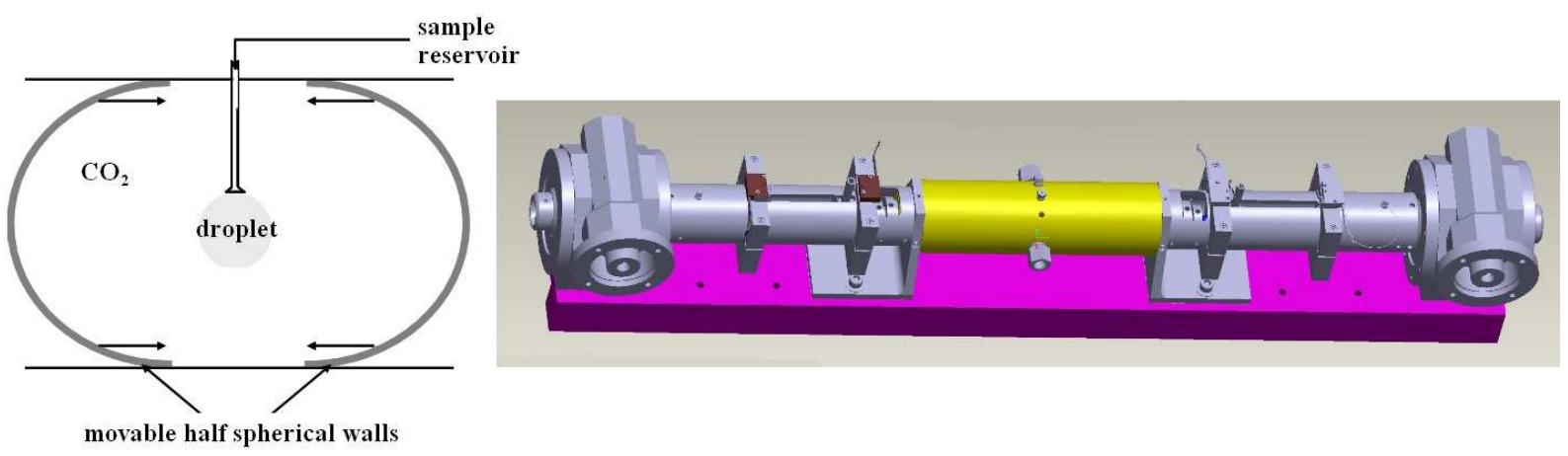

Figure 2.1 Concept for the diffusion experiment (left, side view) and the 3D experiment construction (right) with experiment cell in the middle (the yellow part).

A cylindrical high pressure chamber, as shown in Figure 2.1, with two movable half spherical walls is first filled with sub-critical carbon dioxide. In the middle of the chamber a droplet of sample is produced and fixed (see Figure 3.1, left). The temperature inside the chamber is kept at a constant level above the critical temperature of $\mathrm{CO}_{2}\left(31^{\circ} \mathrm{C}\right)$. By reducing the volume of the chamber (increasing the pressure while compressing), $\mathrm{CO}_{2}$ reaches its supercritical state and the dissolution process of the sample is optically observed. As the phase transition is very sensitive to the small changes in pressure and in order to obtain measurements with high accuracy, the concept aims to eliminate the density driven convection, that would affect the pressure distribution inside the chamber. As the sample is a droplet, the chamber walls are specially designed in half spherical shape, to minimize the induced flow during a compression. This way, the direction of the change in density is constrained to a direction perpendicular to the droplet surface and the variations in concentration fields near the droplet are restricted to a minimum. 
In the experimental setup the volume of the cell was controlled with two pistons on the sides, which were driven by two servo motors (see Figure 2.1, right). The experiment system was later assembled in two different configurations to meet the safety requirements of a parabolic flight campaign as well as to achieve a better performance of the setup in earth laboratory, respectively.

\subsection{System description}



Figure 2.2 Experiment system plan, with "OV" indicating overpressure valves, "BV" burst plate valves and "MV" manual valves.

The experiments took place in the experiment cell - the high pressure compressor which enabled an optical access during experiment. Besides the three accesses to the experiment cell (for medium injection $\left(\mathrm{CO}_{2}\right)$, sample injection and waste outlet), on the cell wall there were also mounting holes for the temperature as well as pressure sensors, which served to control and monitor the experimental conditions.

\subsubsection{High pressure system}

The experiment cell can hold a pressure up to 150bars, which can be achieved by isothermal compression of the cell. The sample droplet was injected by a HPLC pump with an accuracy of flow control down to $0.001 \mathrm{ml} / \mathrm{min}$. The high pressure system consisted of three manual valves (see Figure 2.3 below) with the purpose to reduce the dead volume inside the high pressure capillaries. To fulfill the safety requirements for such high pressure systems in parabolic flight campaign, two overpressure valves (OVs, with an opening pressure of 150bars) and two burst plate valves (BVs, with a burst pressure of 100bars) were also installed into the system according to the "two-fault-tolerance" rule.

In the configuration of the experimental setup in earth laboratory the safety valves (OVs and BVs) were dissembled to further minimize the dead volume during experiments to improve the experiment conditions. 


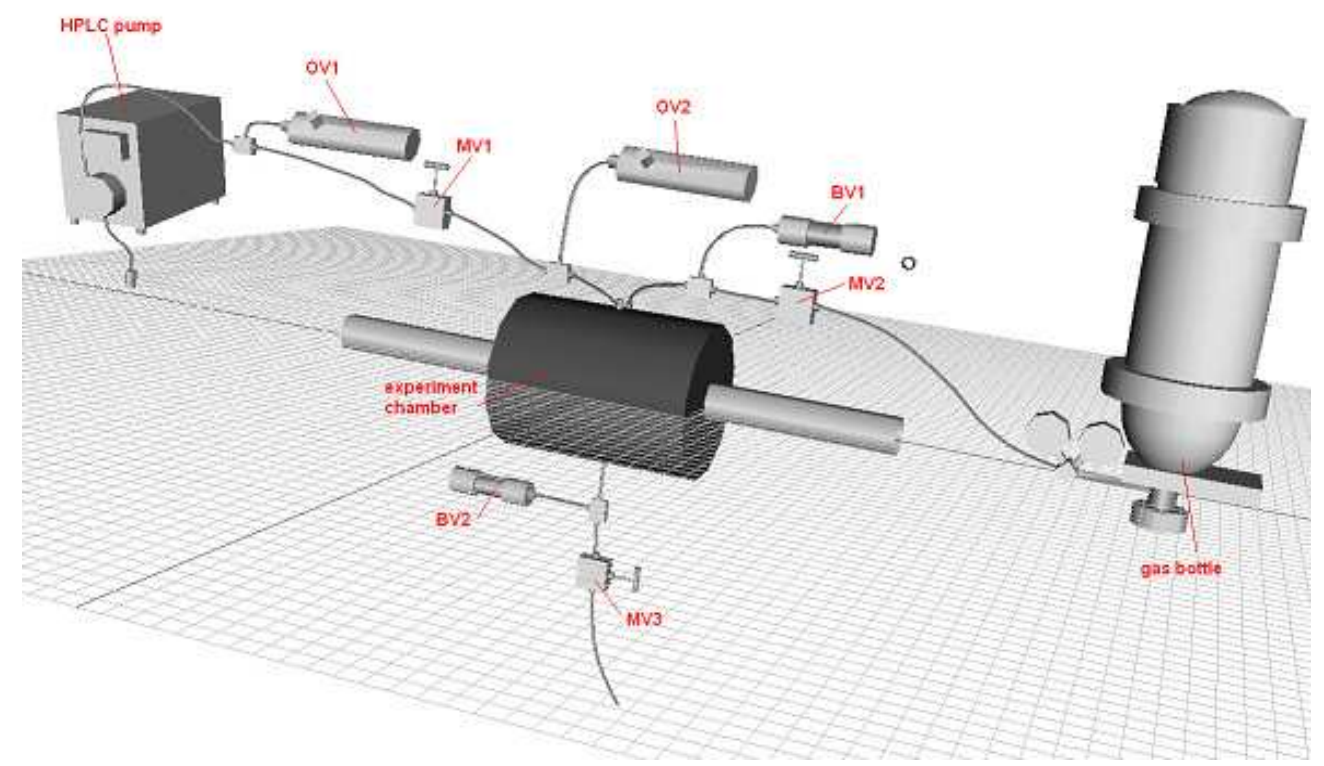

Figure 2.3 The high pressure system (3D demonstration), with the "OV" indicating overpressure valves, "BV" for burst plate valves und "MV" for manual valves.

\subsubsection{Optical system}

During an experiment run the pressure and the temperature inside the experiment chamber were monitored and registered, and the observation of the diffusion process was accomplished by means of a shearing interferometer with a $12 \mathrm{~mW}$ He-Ne-Laser (wavelength of $632.8 \mathrm{~nm}$ ), two Wollaston prisms as the shear-unit and a high speed camera as detector (with $1024 \times 1280$ pixels and $12 \mu \mathrm{m} \times 12 \mu \mathrm{m} /$ pixel, with a max. frame rate of $636 \mathrm{fps}$ ), see Figure 2.4 below. The "infinite fringe alignment" - arrangement was applied for the measurements.



Figure 2.4 Shearing interferometer setup. "M" indicates mirrors, "P" for polarizers and "WP" for Wollaston prisms.

This construction was under the consideration of experiment conditions in flight as well as the actual space in the experiment rack, in the configuration in earth laboratory after flight experiments unnecessary components (e.g. multiple mirrors, etc.) were dissembled, which would bring in additional errors to the system. 


\subsubsection{Mechanical and electrical system}

Optical methods especially those concerning lasers are well known for their accuracy and at the same time their sensitivity to their mechanical constructions, i.e. their own fixations, the vibrations during experiments, etc. Considering the experiment conditions in an airplane, the shearing interferometer setup was chosen because of its satisfying stability agsainst strong mechanical vibrations which were not avoidable. According to the different requirements in flights as well as in earth laboratory two mechanical configurations were constructed (see Figure 2.5 below) to guarantee the proper experiment functionality of the whole system.
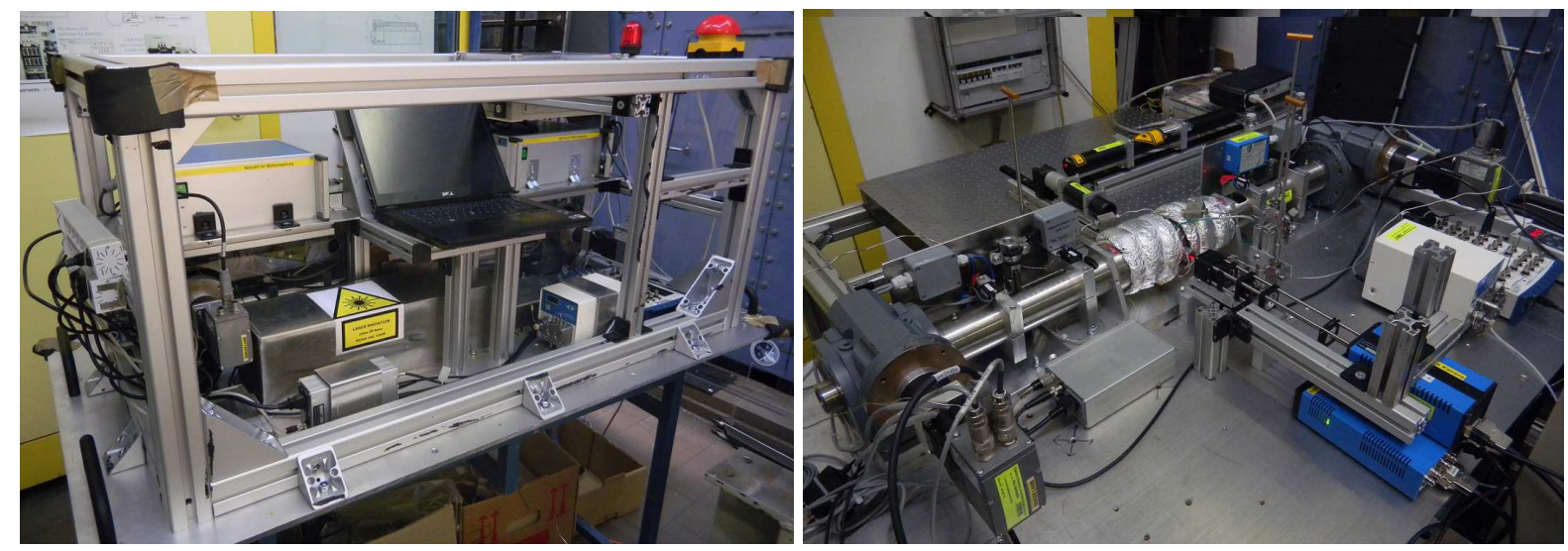

Figure 2.5 The experiment configurations for parabolic flight campaign (left) and in earth laboratory (right), respectively.

For experiments in flight the configuration was safety-orientated with double-containment-rule that all laser components were covered with protection plates and laser adsorbing material was attached on the inside. In the configuration in earth laboratory the laser components were assembled on a standard optical bank to better assure the maximum measurement capacity of the whole setup.

Electrical systems are mainly data acquisition systems, which consist of temperature-/pressure acquisition and the image acquisition from the interferometer (also: interferogram), as well as the motor control system. For the flight configuration a fuse box was built in to meet the safety requirement.

The technical data of the equipment used in the experiments are summarized in Table 2.1 below.

\begin{tabular}{|l|l|l||l||l|}
\hline & $\begin{array}{l}\text { Temperature } \\
\text { sensor }\end{array}$ & Pressure sensor & He-Ne-Laser & High speed camera \\
\hline Sample rate & $40 \mathrm{~Hz}$ & $40 \mathrm{~Hz}$ & Wavelength: $632.8 \mathrm{~nm}$ & $\begin{array}{l}\text { Resolution: 1280 } \mathrm{x} \\
1024 \text { pixels }(12 \mu \mathrm{m} x \\
12 \mu \mathrm{m}) \text { with } 1 \quad \mathrm{x} \quad 1 \\
\text { binning, max. 636fps }\end{array}$ \\
\hline $\begin{array}{l}\text { Measurement } \\
\text { range }\end{array}$ & $0-200^{\circ} \mathrm{C}$ & $0-250 \mathrm{bars}$ & Power: $12 \mathrm{~mW}$ & \\
\hline
\end{tabular}

Table 2.1 Technical data of the equipment used in the experiment.

\section{Experiment plan and setup calibration}

In the overall experiments three test-oil samples were used - rose oil (an aromatic oil for household as air fresher, commercial use), a lubricant oil (Croda, a gear oil in automobiles, commercial use) and DL-alpha-tocopherol (Merck, 99.8\% purity, research use only). The three test-oils exhibited increasing density and viscosity. And after the pretests, they had been proved to be able to demonstrate three different scales of transport phenomena characterized by different diffusion velocities (diffusion 
coefficient, i.e.) into the solvent, which were directly observable from the fringe patterns of the interferograms.

The experimental conditions were first set to assimilate the conditions that usually encountered in the industrial procedures of supercritical fluids processing, i.e. the temperature was near room temperature $\left(35-45^{\circ} \mathrm{C}\right)$ and the pressure was adjustable in a relatively wide range $(60-100 \mathrm{bars}$, limited by the capacity of the experiment cell). The relatively small dimensions of the experiment cell defined the usable amount of solvent, and therefore limited the dissolubility of the solute in this certain amount of solvent. In order to obtain the most advantageous observation a wider range of temperature was set in the later experiments. The overall experiment region is illustrated in Figure 3.1 below.

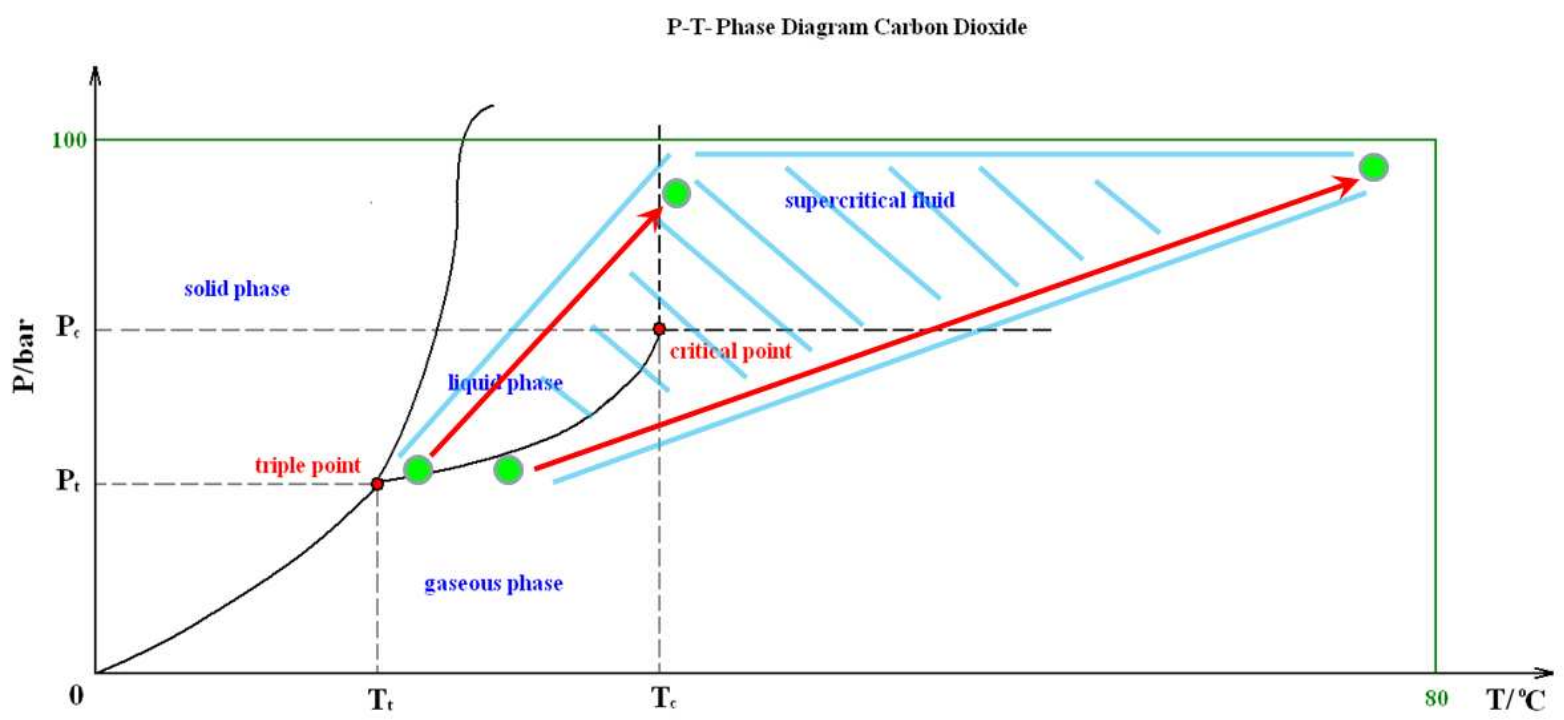

Figure 3.1 Experiment region illustrated in a P-T-phase diagram.

The experimental setup requires a proper validation and scale up so that with direct observations of the interferograms in real-time, one can conveniently categorize the sample oil into the correspondent property class. This can be realized by testing the reference-oil samples with the experiment setup, whose transport properties were well studied and reported in the literatures. As the first tests, in order to obtain obvious observations under relatively low temperature condition, some substances with higher diffusion coefficients were chosen - acetone, benzene and toluene, with increasing molecular weight as well as density. In addition, samples as naphthalene and oleic acid methyl ester were taken as alternatives (see Table 3.1 below). Depending on the results, further reference-oil samples with smaller diffusion coefficients would be applied.

The diffusion coefficients demonstrated in the table above were taken from the data provided by different authors for the same substance with different measurement methods ([5] [6] [7]). Some of them show a nonlinear behavior with increasing pressure at a constant temperature.

Besides diffusion phenomena it is also of interest to observe the dynamic of the fluid behavior in its supercritical state as well as during the phase transition. For this purpose isothermal compression and expansion were also carried out. 


\begin{tabular}{|l||l|l||l|l|l|}
\hline & $\begin{array}{l}\text { Molar weight } \\
{[\mathrm{g} / \mathrm{mol}]}\end{array}$ & $\begin{array}{l}\text { Density } \\
{\left[\mathrm{kg} / \mathrm{m}^{3}\right]}\end{array}$ & Temperature [K] & Pressure [bar] & $\begin{array}{l}\text { Diffusion } \\
\text { coefficient } \\
{\left[10^{-8} \mathrm{~m}^{2} \mathrm{~s}\right]}\end{array}$ \\
\hline \hline Acetone & 58.08 & 792.5 & $303-333$ & $110-160$ & $1.4-2.7$ \\
\hline Benzene & 78.11 & 876.5 & $303-333$ & $110-160$ & $1.3-2.5$ \\
\hline Toluene & 92.14 & 866.9 & $313-373$ & 100 & $1.5-4.5$ \\
\hline Naphthalene & 128.17 & 1140 & $303-333$ & $72-90$ & $3.8-1.22-1.1$ \\
\hline $\begin{array}{l}\text { Oleic acid } \\
\text { methyl ester }\end{array}$ & 296.5 & 870 & 313 & $80-110$ & $1.7-1.1-0.8$ \\
\hline
\end{tabular}

Table 3.1 Properties of reference-oil samples.

\section{Results and discussions}

\subsection{Calibration}

Calibration experiments with the reference-oil samples (acetone, benzene, toluene, etc.) were carried out in earth laboratory, the experiment temperature was first set to a lower value $\left(35-40^{\circ} \mathrm{C}\right)$ and the pressure was varied between ca. 65bar and 90bar. Example images of fringe patterns in the interferograms are shown in Figure 4.1 below.
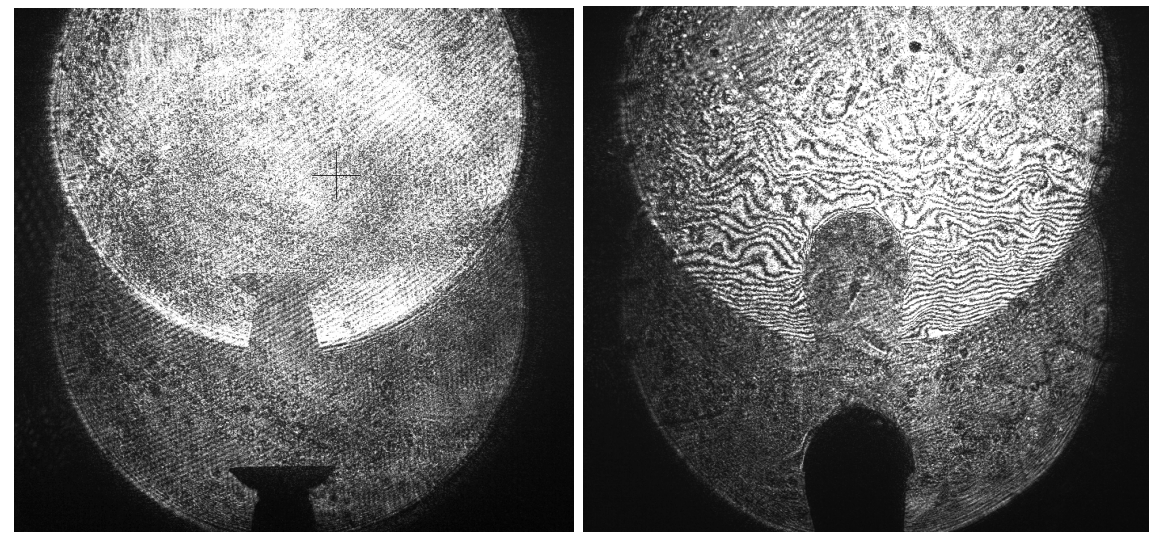

Figure 4.1 Example images of interferometer, after laser adjustment - infinite fringe alignment (left) and experiment with acetone droplet in earth laboratory (right, 308K and 78bar).

Experiments with the reference-oil samples (acetone, benzene, toluene, etc.) were carried out in earth laboratory and processed. The characteristics of the interferograms, e.g. number and width of the fringes, the distances between the fringes around the droplet, additionally also the changes of the droplet size in time were registered as references. Also for the purpose of setting the references of the results, the outlet process of the medium from the experiment cell through the sample injection line were also captured and processed, where the changes of the velocity field (i.e. the density changes) observed were easily predictable by aerodynamic relations for a pressure balancing process. Example images for an outlet process are shown in Figure 4.2 below.

\subsection{Results from the earth laboratory}

The diffusion phenomena were observed in different time scales of ca. $1.5 \mathrm{~s}$ for the rose oil and ca. $15 \mathrm{~s}$ for the lubricant oil, respectively. In the case of tocopherol which has a typical observable diffusion time of about $30 \mathrm{~min}$ to one hour, no simultaneous density changes were observed besides the changes 
of the sample droplet's size. All experiments were carried out under isothermal condition under different pressures, i.e. the medium was heated to a defined temperature, which was kept constant while the pressure was adjusted to new values - isothermal compression and expansion.

Figure 4.3 below shows two example image sequences of rose oil and lubricant oil with the most obvious observations of density changes occurred at ca. $333 \mathrm{~K}$ and $75 \mathrm{bar}$.


Figure 4.2 Example images of an outlet process (through the injection capillary in the middle) as reference for density changes in the medium, with a start pressure at ca. $65 \mathrm{bar}$.

Comparing the two samples, rose oil had the smaller viscosity and diffused faster (density changes in ca. $1.5 \mathrm{~s}$ compared to lubricant oil in ca. 15s), which could be directly evaluated from the interferograms.

\subsection{Observation under microgravity}

One of the goals of the project is the observation of diffusion under microgravity to avoid the influence of the gravity-driven convection that overlaps the diffusion process on earth. For the flight experiments in the most recent campaign ( $54^{\text {th }}$ ESA parabolic flight campaign) only tocopherol as sample was certified, so no significant changes of the droplet as well as in the medium concerning a diffusion process was observed. Example images of an arbitrary parabola during the flight campaign are shown in Figure 4.4 below.

From the recordings of the flight experiments it can be seen that the microgravity phase stabilized after the first $3-5 \mathrm{~s}$. Image 4 is the most optimal condition for the observation of a convection free diffusion process.

Other goals of the flight campaign were to test the recording of the behavior of $\mathrm{SCCO}_{2}$ in microgravity phase and to successful carry out the experiment procedures (droplet injection in both $0 \mathrm{~g}$ and $2 \mathrm{~g}$ phases, experiment cell volume control by driving the pistons, etc.). The functionality of the experiment setup was proved and confirmed to be able to provide a stable environment for observation of diffusion also under microgravity conditions. 



Figure 4.3 Example image sequences from experiments with rose oil (left, in ca. 1.5s) and lubricant oil (right, in ca. 15s), respectively.

Gravity data-parabola31-27.05.2011
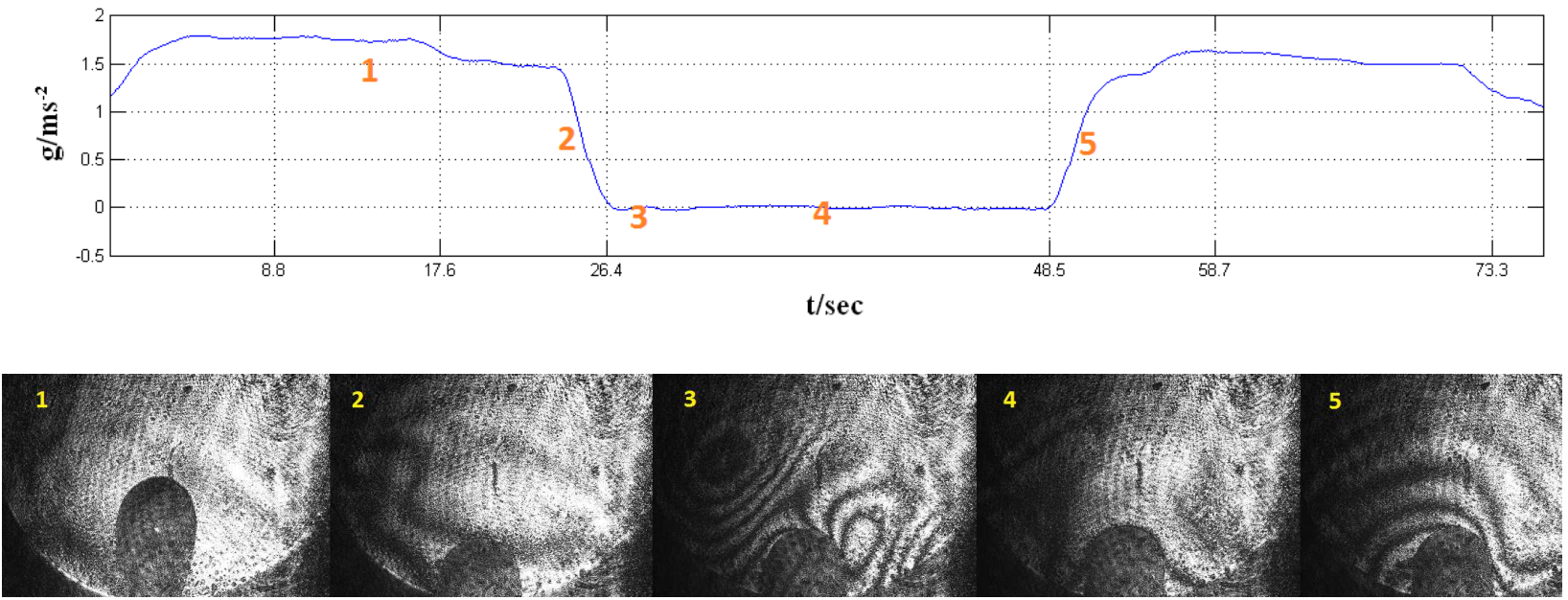

Figure 4.4 One image sequence for a parabola, images 1 to 5 were from ca. $2 \mathrm{~g}$ to $0 \mathrm{~g}$. 


\section{Data processing and model fitting}

\subsection{Interferogram analysis}

A shearing interferometer links the variations in refractive index gradients observed as fringe shifting in an interferogram to the variations in density gradients, with one fringe shift corresponding to one wavelength $\lambda$ of the laser used by

$$
\frac{\Delta l}{\lambda}=\frac{\Delta S}{S} \text {. }
$$

with $\Delta l$ being the optical length change, $\Delta S$ the fringe shifting and $S$ the fringe distance before shifting. Under the assumption that the test object (the experiment cell) is a 2-D object (considering the $12 \mathrm{~mm}$ distance between the windows, and the ca. $2 \mathrm{~mm}$ sample droplet), refractive index, i.e. the density, does not vary in the laser propagation direction, the optical length change $\Delta l$ of the laser beam after passing the test object is obtained by

$$
\Delta l(x, y)=d \cdot \int_{z_{1}}^{z_{2}} \frac{\partial n(x, y)}{\partial \xi} d z=d \cdot \frac{\partial n(x, y)}{\partial \xi} \cdot L
$$

with

$d$ - the shear-length of the interferometer setup,

$n$ - the refractive index,

$L$ - the length of the test object in the laser propagation direction,

$\xi$ - the integration direction.

The Clausius-Mosotti relation [8], which links the refractive index to density, yields

$$
\frac{n^{2}-1}{n^{2}+2}=\frac{2}{3} K \cdot \rho
$$

where,

$\rho$ - the density,

$K$ - the Gladstone-Dale constant.

Combination of Eq. (1) and (2) leads to

$$
\frac{\partial n}{\partial \xi}=\frac{\lambda}{d \cdot L} \cdot \frac{\Delta S}{S} .
$$

With the following form the density gradients are obtained

$$
\frac{\partial \rho}{\partial \xi}=\frac{\partial \rho}{\partial n} \cdot \frac{\partial n}{\partial \xi}
$$

To calculate the diffusion coefficient Fick's $1^{\text {st }}$ Law [9]

$$
J=-D \frac{\partial c}{\partial \xi}
$$

is applied, where, 
$J$ - the flux,

$D$ - the mutual diffusion coefficient,

$c$ - the mole concentration of the sample.

It is assumed that the diffusion coefficient is constant for each image in a sequence and that there are only small concentration changes between images (adjustment by setting the delay between the images) compared to the whole process. As in the experiments the droplet injection was carried out in a continuous mode, the surface concentration of the droplet remains unchanged. According to mentioned criteria the following relation is obtained,

$\frac{\partial \rho}{\partial t} \cdot \Delta s_{i}=D_{i} \cdot \frac{\partial \rho}{\partial \xi}$

with, $\Delta s_{i}-$ the diffusion length in each image,

$\rho$ - the density of the medium,

$i$ - the image number in one sequence.

For all images in a sequence a diffusion coefficient is calculated by analyzing the fringe shiftings (see Figure 5.1).
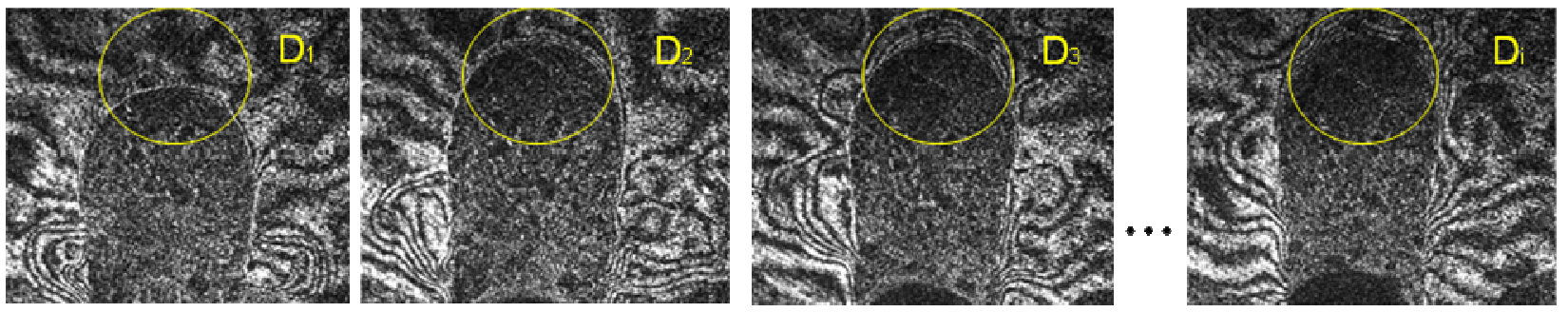

Figure 5.1 The diffusion coefficient was calculated for each image in one certain area. Due to the vertical shear (highest sensitivity) the top of the droplet was chosen.

\subsection{Evaluation of the droplet size}

When the test-oil samples had a relatively small diffusivity (by ca. $10^{-9} \mathrm{~m}^{2} / \mathrm{s}$ ) at the moderate temperature, or after many injections of the oil samples the solvent had almost reached its saturation limit, no obvious fringe patterns in the interferograms could be observed. In these situations the data processing could be accomplished by evaluating the changes of the droplet size during an isothermal compression. An example is shown in Figure 5.2 below.
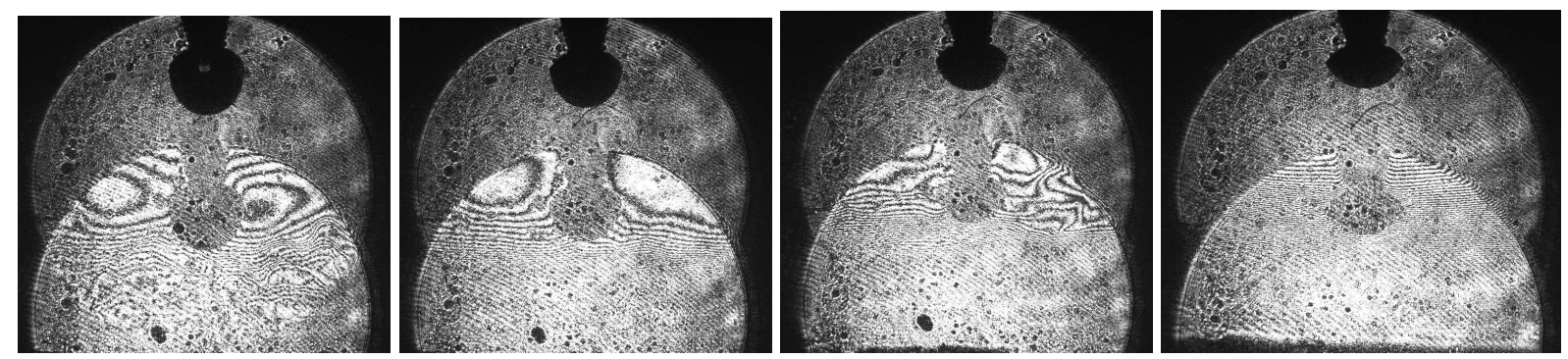

Figure 5.2 Example images of solute dissolution during an isothermal compression, which enabled the evaluation of the transport property by analyzing the droplet size changes in time. 
The requirement of this approach was to properly choose the compression in a relatively small pressure range (within 5bars) to make sure that the diffusivity could be assumed constant and at the same time an optimal size change could be captured.

By applying the Fick's first law for diffusion in a radial symmetrical geometry:

$\frac{\partial c}{\partial t}=D \cdot \frac{1}{r^{2}} \cdot \frac{\partial}{\partial r}\left(r^{2} \cdot \frac{\partial c}{\partial r}\right)=D \cdot \frac{1}{r^{2}} \cdot\left(2 r \frac{\partial c}{\partial r}+r^{2} \frac{\partial^{2} c}{\partial r^{2}}\right)$

with

$c$ - the mole concentration of the solute,

$t$ - the diffusion time (compression time),

$D$ - the diffusion coefficient of the solute,

$r$ - the integration direction.

Considering the dimension and condition of the experiment, that the droplet disappears completely in the end, the following relation is valid,

$\frac{\partial c}{\partial r} \approx \frac{c_{0}}{R}$

(9) and

$\frac{\partial c}{\partial t} \approx \frac{c_{0}}{T}$

while

$c_{o}-$ the initial mole concentration,

$R$ - radius of the droplet,

$T$ - the experiment time.

By means of equations (9) and (10) together with (8) the following relation can be obtained,

$\frac{c_{0}}{T} \approx D \cdot \frac{1}{R^{2}} \cdot\left(2 \cdot R \cdot \frac{c_{0}}{R}+R^{2} \cdot \frac{c_{0}}{R^{2}}\right)=D \cdot \frac{1}{R^{2}} \cdot 3 \cdot c_{0}$.

So the diffusion coefficient could be calculated by

$D \approx \frac{1}{3} \frac{R^{2}}{T}$

\subsection{Semi-empirical diffusion model based on experiment data}

A nonlinear diffusion model was applied and all the parameters were chosen in order to approximate the experiments. According to this fitted model the diffusion process was simulated.

Nonlinear diffusion is a further approach to the real diffusion situation, in which the diffusion coefficient varies with the density gradient. According to the Fick's $2^{\text {nd }}$ Law, 
$\frac{\partial c}{\partial t}=\frac{\partial}{\partial \xi}\left(D_{n l} \cdot \frac{\partial c}{\partial \xi}\right)$

where $D_{n l}=D_{n l}\left(\frac{\partial c}{\partial t}\right)$ is the function of the concentration gradient,

$c$ - the mole concentration of the medium,

$D_{n l}$ - nonlinear diffusion coefficient, which can be describe by the following form,

$D_{n l}=1-\exp \left(-\frac{C_{m}}{(g / \lambda)^{m}}\right)$ for $g>0$ and $D_{n l}=1$ for $g<=0$.

The parameters $C_{m}, \lambda$ and $g$ are chosen according to the experimental observations and conditions as well as considering the dependence between the three.

The diffusion calculated with the model is displayed in Figure 5.3 below.

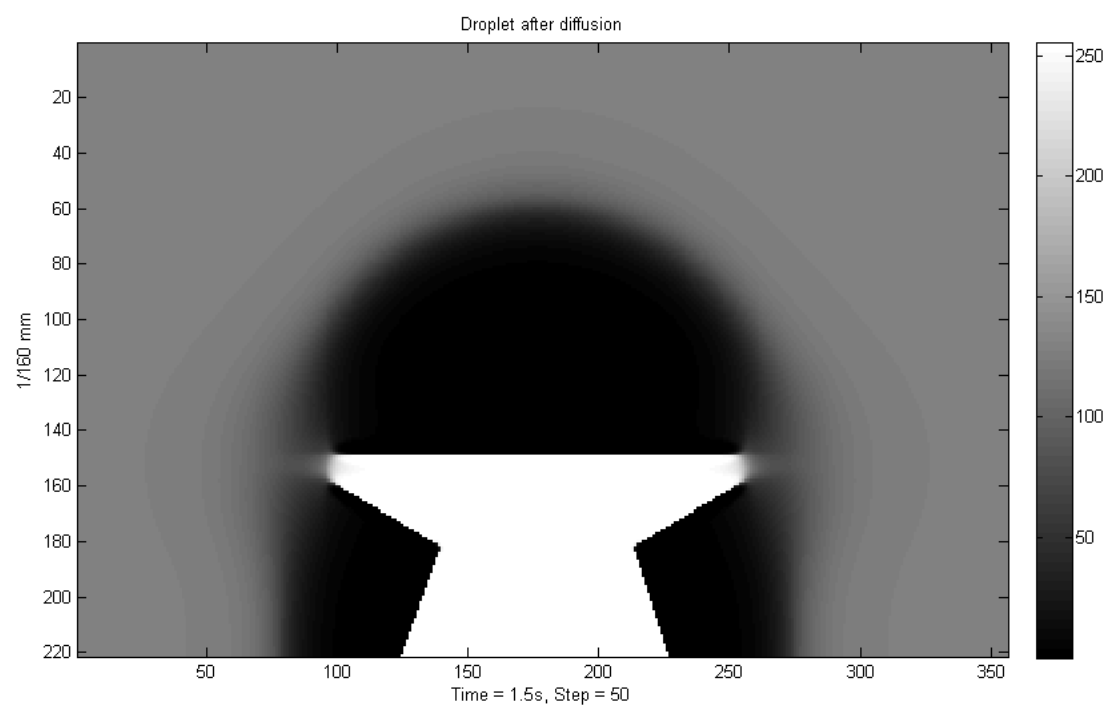

Figure 5.3 The diffusion layers were calculated according to the experiment conditions (rose oil) as well as considering the form of the injection capillary (in the middle) and the real density difference between the droplet and the medium. [10]

After a nonlinear diffusion process a variation of the diffusion velocity with time was observed and the diffusion fronts were mainly the propagation of the contour lines of the droplet (which were the same as the fringes in an interferogram). The deviations from the droplet contour were observed both in the model and in the experiment at the sharp edges of the injection capillary, where the density gradient varies faster than that in the other regions. This phenomenon in the experiment is shown in Figure 5.4 below. 

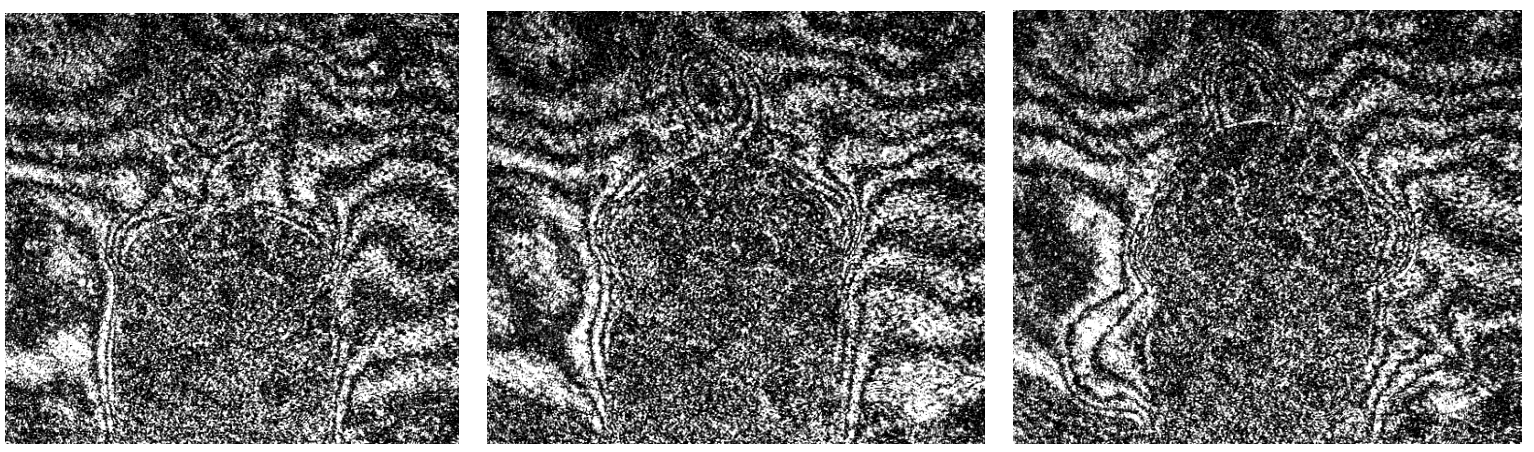

Figure 5.4 The deviation of the diffusion fronts from the droplet contour at the sharp edges of the injection capillary in an image sequence (rose oil).

For experiment results from earth laboratory with the influence of convection the transport process is to be characterized with transport coefficient, instead of diffusion coefficients. The obtained values from one image sequence of rose oil as well as the calculated results from the model were plotted in Figure 5.5 below.

From the plot in Figure 5.5 the aberration between the data set and the model is obvious. The measured diffusivities are generally faster than those from the calculations, as in earth laboratory the dissolving process had been the combination of diffusion and convection. And the fluctuations of the data set indicated that small variations of the density in the bulk medium were not avoidable.

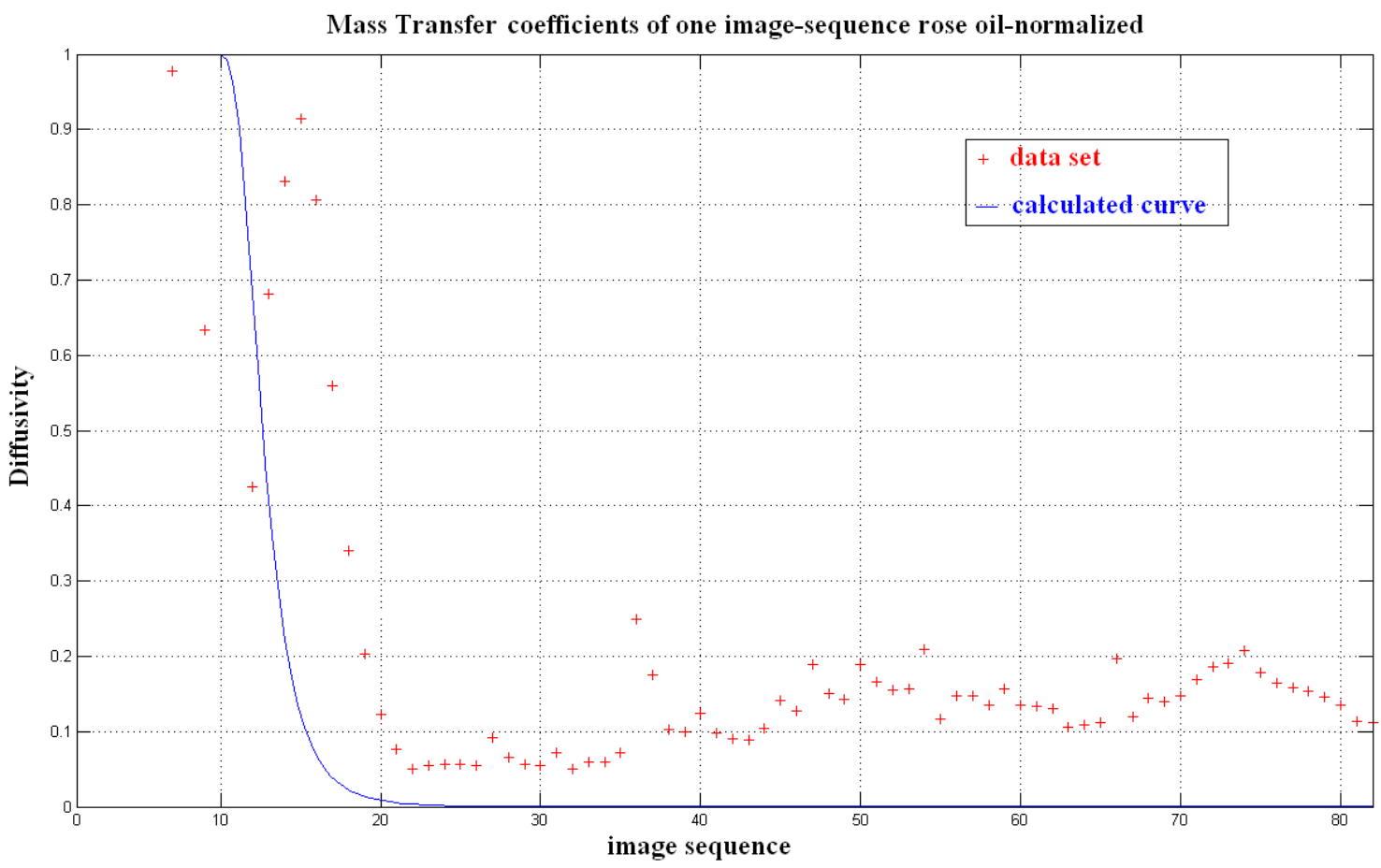

Figure 5.5 Plot of the diffusion coefficients from experiment and from calculation (for better comparison the diffusivity was normalized). 


\section{Conclusions and further aspects}

In order to observe a relatively fast diffusion process (usually in seconds) in compressible fluids interferometry was proved to be a suitable method considering the accuracy and also the simple technical realisation. The only requirement on the experiment setup is the optical access. With the reference tests of the oil samples that have the identified transport properties, the experiment setup can be proper scaled and therefore be well validated. By in situ observation of the interferograms the transport properties if the test-oil samples are to be directly evaluated, the correspondent coefficients can be approximately estimated according to the established catalogue of references.

Besides, the saturation limit of a certain solute under a certain pressure and temperature can also be directly observed from the interferometer, e.g. in an isothermal compression or expansion procedure. With precisely controlled amount of mediums it is also possible to quantitatively estimate the solubility data with the setup, which shows the new applicability of this optical measurement method. An example is shown in Figure 6.1 below.
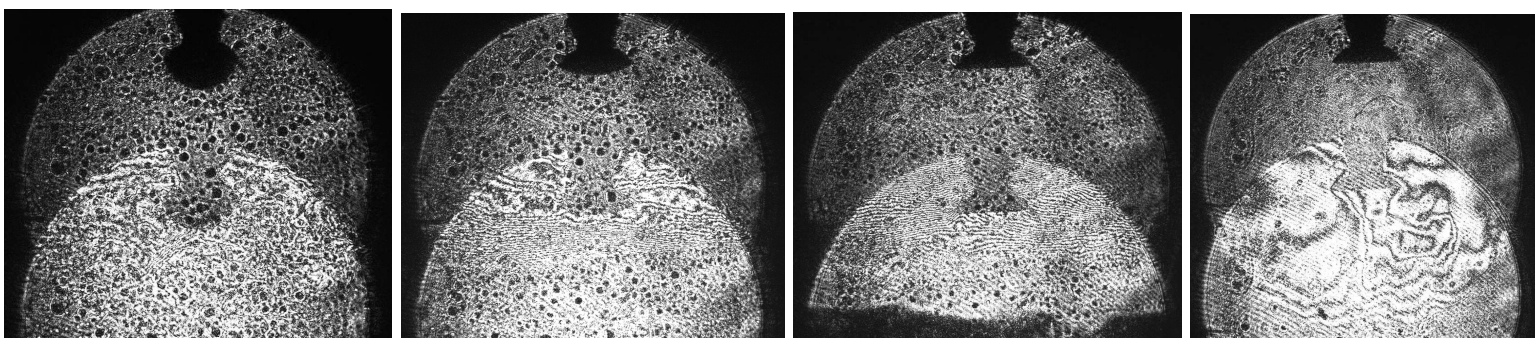

Figure 6.1 Observation of the solute dissolution during an isothermal compression. With the pressure increasing, the solute condensed on the windows gradually dissolved into the medium (lubricant oil, start pressure: 70bar, end pressure: 90bar).

With the interferometer setup besides the dynamic of fluid under various experiment conditions many other phenomena could also be observed, e.g. the transport behaviour of a mixture of oil samples, or the injection of a gas bubble into the liquid medium, which proves the applicability of the experiment setup in the research of numerous different kinds of fluids interactions, as shown in Figure 6.2 below. Considering its easy-to-use advantages and high accuracy comparing to the other measurement methods to obtain material properties, it opens a new aspect of both visualizing and analyzing the thermodynamic processes.
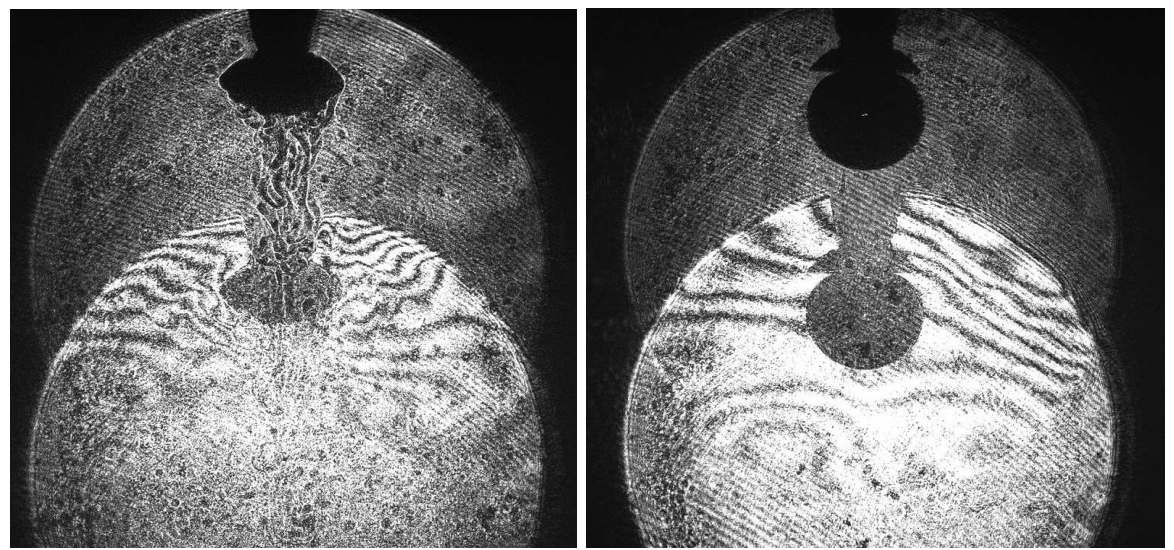

Figure 6.2 Example images of a mixture droplet of rose oil and benzene (left) and the injection of a gas bubble into a liquid reservoir. 
The nonlinear diffusion model can be applied to describe the diffusion process in supercritical fluids. The necessary parameters can be obtained from comparison with the experimental results and later an improvement by generalization for different samples was also possible.

As further aspects, more experiments under compensated gravity are desired with possibly more testoil samples in order to obtain enough data to be compared with the results from the earth laboratory. The data analysis was accomplished analytically until now. A further approach is to use numerical methods, which also can be applied to model fitting and further process simulations. From the observation and evaluation of the interferograms (i.e. acquisition of transport properties) obtained from experiments it also opens the opportunity to characterize the interactions of solute and solvent particles, which will provide necessary feed backs for simulations at molecular level.

\section{References}

[1] Arai Y, Sako T and Takabayashi Y (Eds.) 2002 Supercritical Fluids, Molecular Interactions, Physical Properties and New Applications (Springer-Verlag Berlin und Heidelberg)

[2] Mohamed R S and Mansoori G A June 2002 Featured Article - Food Technology Magazine The World Market Research Centre (London UK)

[3] Stahl E and Quirin K. W 1983 Fluid Phase Equilibria 8 p 93

[4] Liong K K, Wells P A and Forster P R 1991 Journal of Supercritical Fluids 4 p 91

[5] Sassiat R, Mourier P, Caude M H and Rosset R H 1987 Analytical Chemistry 59 p 1164

[6] Dahmen N, Kordikowski A and Schneider G M 1990 Journal of Chromatography 505 p 169

[7] Higashi H, Iwai Y, Takahashi Y, Uchida H and Arai Y 1998 Fluid Phase Equilibria 144 p 269

[8] Merzkirch W 1987 Flow Visualization (2. Ed., Academic Press, New York and London)

[9] Crank J 1985 The mathematics of diffusion (2.Ed. Oxford Science Publications, Clarendon)

[10] Nonlinear Diffusion Toolbox ( Mathworks Inc.)

\section{Acknowledgements}

The project is funded by DLR (German Aerospace Agency). The authors would like to thank both DLR and ESA for the opportunity of conducting experiments in the two parabolic flight campaigns ( $16^{\text {th }}$ DLR and $54^{\text {th }}$ ESA parabolic flight campaign). 